\title{
Questes
}

Revue pluridisciplinaire d'études médiévales

\section{Actum Rome: les pratiques d'authentification des actes notariés à Rome à la fin du Moyen Âge}

Cécile Troadec

\section{OpenEdition}

Journals

Édition électronique

URL : http://journals.openedition.org/questes/3574

DOI : 10.4000/questes.3574

ISSN : 2109-9472

Éditeur

Les Amis de Questes

Édition imprimée

Date de publication : 15 janvier 2015

Pagination : 137-154

ISSN : 2102-7188

\section{Référence électronique}

Cécile Troadec, «Actum Rome : les pratiques d'authentification des actes notariés à Rome à la fin du Moyen Âge », Questes [En ligne], 29 | 2015, mis en ligne le 24 juin 2015, consulté le 19 avril 2019. URL http://journals.openedition.org/questes/3574 ; DOI : 10.4000/questes.3574 


\title{
Actum Rome : les pratiques d'authentification des actes notariés à Rome à la fin du Moyen Âge.
}

\author{
Cécile TROADEC \\ Université Paris-Sorbonne - Università degli Studi Roma Tre
}

Le 24 juillet 1460, dans un registre du notaire romain Antonius de Thomeys, est rédigé un acte singulier qui atteste qu'une petite fille de six ans, Paulina, a perdu sa virginité en tombant dans l'escalier de la maison familiale ${ }^{1}$ : la fillette est examinée par un aréopage de femmes qui atteste devant témoins de son état physique. Le notaire produit alors un texte, qui vise à prémunir la petite Paulina contre tout différend qui pourrait survenir au moment de son mariage. Les étapes de l'authentification de cet événement sont plurielles, et l'écrit notarié vient les couronner et les verrouiller.

$\mathrm{Au} \mathrm{XI}{ }^{\mathrm{e}}$ siècle à Rome, les notaires (scriniarii) supplantent rapidement les tabelliones de l'Antiquité en tant que rédacteurs privés ${ }^{2}$. Le statut du notaire romain, comme du reste l'ensemble du notariat italien, connut, au cours du XII ${ }^{\mathrm{e}}$ siècle, une mutation majeure. De simple scribe, il devient un officier institué par une autorité publique

Il possède désormais la capacité - symbolisée par

l'invention du signum - de conférer un caractère

\footnotetext{
${ }^{1}$ Roma, Archivio di Stato di Roma (ASR), Collegio dei Notai Capitolini (CNC), 1774, notaire Antonius de Thomeys, $\mathrm{f}^{\mathrm{0}} 74 \mathrm{r}^{\circ} \mathrm{v}^{0}, 24$ juillet 1460.

${ }^{2}$ Cristina Carbonetti, " Gli scriptores chartarum a Roma nell'alto medioevo », dans Notariado publico y documento privado : de los origenes al siglo XIV, actas del VII Congreso internacional de diplomatica, dir. José Trenchs Odena, Valencia, Conselleria de cultura, educacio I ciencia, 1989, vol. 2, p. 1109-1137.
} 
authentique à ses écritures, préparatoires (minute ou brève) ou mises au net (mundum, instrument) ${ }^{3}$.

C'est cette fides publica, le plus souvent conférée par une autorité impériale, qui fonde le caractère probatoire des actes notariés, lesquels peuvent être produits comme preuves lors d'un procès. Au début de chaque registre, le notaire rappelle toujours la source de sa légitimité, plaçant sous l'égide du pape ou de l'empereur l'ensemble des actes qui suivent. Ainsi, Evangelista de Bistuciis ${ }^{4}$ ou Augustinus de Martinis ${ }^{5}$ se déclarent «civis romanus Dei gratia publicus imperiali auctoritate notarius ».

Dans un contexte politique et social troublé, le recours au notaire est de plus en plus fréquent dans la Rome du $X^{\mathrm{e}}$ siècle, même lorsque le montant de la transaction est modique. Le collège des notaires romains devient pléthorique, et la masse de minutes notariées, désormais rédigées sur papier, est de plus en plus importante. En période d'instabilité, le notaire apparaît comme une figure d'autorité, à laquelle on peut se fier pour garantir la régularité des transactions économiques et la paix sociale ${ }^{6}$. En réponse à ce besoin de stabilité et d'authenticité, les notaires romains mettent en œuvre de nouveaux modes de validation de leurs actes afin d'en garantir l'originalité.

3 Jean-Louis Gaulin, «Affaires privées et certification publique. La documentation notariale relative au crédit à Bologne au XIII ${ }^{\mathrm{e}}$ siècle », dans Notaires et crédit dans l'Occident méditerranéen médiéval, dir. François Menant et Odile Redon, Rome, École française de Rome, 2004, p. 55-95.

${ }^{4}$ Roma, ASR, CNC, 1896, notaire Evangelista de Bistuciis, $\mathrm{f}^{\circ} 19 \mathrm{r}^{\circ}$.

${ }^{5}$ Roma, ASR, CNC, 1082, notaire Augustinus de Martinis, $\mathrm{f}^{\circ} 42 \mathrm{r}^{\circ}$.

6 Anna Maria Corbo, «Relazione descrittiva degli archivi notarili romani dei secoli XIV-XV nell'Archivio di Stato e nell'Archivio Capitolino », dans Gli atti privati nel tardo medio evo : fonti per la storia sociale, dir. Paolo Brezzi et Egmont Lee, RomaToronto, Istituto di studi romani, p. 49-69, p. 50: «soprattutto tra la metà del secolo XIV e la metà del XV, periodo in cui, nella instabilità sociale e politica, si faceva riferimento al notaio come autorità costante alla quale affidare la certezza dei rapporti giuridici tra cittadini ». 


\section{De la minute à l'instrument, ou comment un document original peut être la matrice de copies originales}

Les pratiques notariales donnent lieu à la rédaction de plusieurs versions successives du texte: d'abord sous forme de notes sur feuille volante, l'acte est ensuite abrégé dans le minutier du notaire. C'est à partir de ce registre que le notaire est à même de produire une ou plusieurs copies authentiques, qu'il remet à son client, et qui pourront servir de preuve juridique ou être conservées par le client dans ses propres archives privées.

La première étape de la confection de l'acte notarié est la rogatio: devant témoins, après que les contractants lui ont confié leur volonté, le notaire rédige une brève note (la notula) qui reprend les données essentielles du contrat (date, noms des parties contractantes et des témoins, nature et clauses du contrat). Dans un deuxième temps, qui suit de peu le moment de la rogatio, le notaire enregistre dans un minutier le document avec ses clauses et la souscription. Conservé dans les archives du notaire, le texte est dès lors doté d'une pleine valeur probatoire. De manière assez surprenante, ce moment de l'imbreviatura, de rédaction de la brève, peut lui-même connaître plusieurs phases, plus ou moins développées. Il peut arriver en effet qu'un même acte fasse l'objet de plusieurs copies au sein d'un même registre notarié, la première étant un brouillon, la suivante une mise au net: ainsi, chez le notaire Augustinus de Martinis, un acte de fiançailles, très raturé aux folios 59-60, est recopié, mis au propre, dans les folios suivants, dans les mêmes termes exactement ${ }^{7}$. Cependant il arrive aussi que le notaire recopie mot pour mot le même acte dans son registre, sans raison apparente ${ }^{8} \ldots$ Ou encore qu'il ne juge pas utile de rédiger la brève et que l'acte en reste au stade initial du brouillon' ${ }^{9}$.

\footnotetext{
${ }^{7}$ Roma, ASR, CNC, 1083, notaire Augustinus de Martinis, $\mathrm{f}^{0} 59 \mathrm{r}^{\circ}-60 \mathrm{v}^{\mathrm{o}}, 16$ décembre 1473 , recopié au propre en $\mathrm{f}^{\circ} 60 \mathrm{v}^{\circ}-63 \mathrm{r}^{\circ}$.

${ }^{8}$ On pourrait citer maints autres exemples, comme cet acte de vente de ruches dans un casale (Roma, ASR, CNC, 1181, notaire Pacificus de Pacificis, $\mathrm{f}^{0} 17 \mathrm{r}^{\circ} \mathrm{v}^{\circ}$, recopié en
} 
Pour expliquer que les pratiques d'écriture et de copie divergent d'un notaire à l'autre, on peut formuler l'hypothèse que certains notaires sont plus soucieux de la memoria et donc de transmettre leurs registres à la postérité : ceux-ci sont attentifs à la bonne tenue des minutiers, à laquelle participe la clarté de la mise en page (utilisation des marges dans lesquelles ils inscrivent un bref résumé du contenu de la minute afin de pouvoir la retrouver plus rapidement en consultant le registre). D'autres notaires, à l'inverse, uniquement préoccupés par l'affaire du moment présent, se contentent d'un brouillon approximatif et raturé, à notre grand dam ${ }^{10}$.

La troisième et dernière étape de la copie est la rédaction, à partir de la minute, de l'instrumentum ${ }^{11}$. Sur demande éventuelle de l'une des deux parties, le notaire rédige sur parchemin l'instrument proprement dit, selon un formulaire bien défini et validé exclusivement par la souscription du rédacteur complétée de son signum. Cette copie peut être conservée dans les archives privées du client. Le contenu de l'acte est le même que celui de la brève, ils contiennent l'un et l'autre les mêmes informations, depuis l'Ars notarie de Raniero de Pérouse (XIII' siècle). En revanche, l'instrument est rédigé entièrement, et non sous une forme abrégée; il est écrit sur

$\mathrm{f}^{\mathrm{o}} 18 \mathrm{r}^{\mathrm{o}} \mathrm{v}^{\circ}$ ); ou encore cette reconnaissance de dettes (Roma ASR, CNC, 926, notaire Iohannes Baptista de Jays, recopiée en $\mathrm{f}^{0} 156 \mathrm{r}^{\circ} \mathrm{v}^{0}$ et en $\left.\mathrm{f}^{0} 158 \mathrm{r}^{\circ} \mathrm{v}^{\circ}\right)$ ou cet acte de vente (Roma, ASR, CNC, 926, notaire Iohannes Baptista de Jays, $\mathrm{f}^{0} 191 \mathrm{r}^{\mathrm{o}} \mathrm{v}^{\mathrm{o}}$, recopié en $\left.\mathrm{f}^{\mathrm{o}} 195 \mathrm{r}^{\mathrm{o}} \mathrm{v}^{\mathrm{o}}\right)$.

9 Voici, à titre d'exemples, quelques actes non rédigés : Roma, ASR, CNC, 1135, notaire Iohannes de Michaelis, $\mathrm{f}^{\circ} 106 \mathrm{r}^{\circ}$ (14 février 1486), $\mathrm{f}^{\mathrm{o}} 109 \mathrm{r}^{\circ}$ (17 février 1486), $\mathrm{f}^{\mathrm{o}} 161 \mathrm{v}^{\mathrm{o}}$ (29 août 1486), f $\mathrm{f}^{\mathrm{o}} 261 \mathrm{v}^{\mathrm{o}}$ (3 juin 1488).

${ }^{10}$ Anna Maria Corbo, « Relazione descrittiva degli archivi notarili romani... », art. cit., p. 56 : «Appare abbastanza chiaro però che solo alcuni di questi notai scrivono per il futuro ; per la storia, e quindi vogliono essere compresi, altri invece sembrano più preoccupati degli affari da concludere ».

${ }^{11}$ Il peut y avoir une quatrième étape de copie, lorsque l'instrument fait l'objet d'une copie qui est conservée dans les archives communales. Cette dernière étape de copie nous échappe totalement à Rome, du fait de la perte des archives communales, mais les statuts de Rome de 1363 y font allusion : la copie enregistrée dans les archives communales doit être contresignée par le juge palatin, avec un degré d'authentification supérieur, et elle vaut dans un procès aussi bien que l'instrument original. Ici l'instrument, de copie, devient l'original qui sert à produire un double. 
parchemin, et non sur papier. Néanmoins, la principale différence au regard de l'originalité du document réside peut-être dans une différence temporelle: il peut s'écouler un grand laps de temps entre la minute et l'instrument, et si la première est rédigée au présent, le second en revanche est rédigé dans un temps du passé. Ce décalage dans la temporalité grammaticale signifie l'immuabilité de l'instrument et surtout sa stérilité : seule la minute est à même de générer des copies, elle seule est la matrice documentaire pour d'autres textes ${ }^{12}$. Il me semble que la différence entre les temps de rédaction est emblématique de cette potentialité propre à l'original qu'est la minute notariée.

La minute est envisagée comme la matrice à partir de laquelle peuvent être produits autant d'instrumenta que requis par le client ou par les autorités communales : alors que cette matrice est le texte premier et original, les copies, pourtant secondes, sont considérées comme de véritables originaux authentiques. La nature du rapport entre original et copie est remarquable : le document initial, texte abrégé et incomplet, est la source même des originaux. Si ces derniers venaient à être perdus ou détruits, ils pourraient être reproduits grâce au minutier. C'est en dernier ressort la minute qui est dotée de la plus forte légitimité : en cas de doute sur la validité d'un instrument produit dans un procès, les statuts urbains prévoient que l'on devra convoquer le notaire et que celui-ci devra produire la minute afin de valider ou d'invalider la copie :

Si aliquis in iudicio produxerit aliquod publicum instrumentum et pars contra quam productum est cum iuramento affirmet et dicat se illud habere suspectum, Senator et Iudex cause ex eorum officio parte petente cogat notarium qui fecit instrumentum et partem producentem ad

12 Armando Petrucci, «Modello notarile e testualità », dans Il notariato nella civiltà toscana : atti di un convegno (maggio 1981), dir. Mario Montorzi, Roma, Consiglio nazionale del notariato, coll. «Studi storici sul notariato italiano », 8, 1985, p. 123145 : « di generare, per copia estratta e svolta, l'originale autentico ». 
exhibendum coram eo prothocolla et abreviaturas dicti instrumenti ut per ea semper imponatur fides et suspectionis allegate tollatur occasio ${ }^{13}$.

C'est pourquoi les autorités publiques ont un grand souci de l'exactitude des informations transcrites par les notaires : la brève comme l'instrument doivent être des copies, des reproductions fidèles de ce que les contractants ont exprimé. Le notaire se doit de ne rien omettre lorsqu'il couche par écrit les propos de ses clients : les statuts de la ville de Rome de 1363 sont particulièrement vigilants sur ce point :

Notarius qui dicta testium examinatorum scribit teneatur substantialiter et explicite scribere dictum testis et non dicat talis dixit ut talis ${ }^{14}$.

Afin de ne pas altérer la véracité de l'acte par oubli ou par erreur le notaire doit, au moment de la rogatio, prendre des notes qu'il mettra rapidement au propre :

Notarius de aliquo contractu vel testamento scribendo rogatus priusquam a loco contractus discedat nomina testium et totam substantiam eius de quo rogatur in quaternutio prothocollorum suorum scribat, ut per oblivionem veritas non mutetur dummodo sibi de suo labore satisfiat ${ }^{15}$.

13 Statuti della città di Roma, éd. Camillo Re, Roma, Tip. della Pace, Biblioteca dell'Accademia storico-giuridica, 1, 1880, Livre I, Rubrique XXXV, «De suspitione instrumenti » : «Si au cours d'un procès quelqu'un produit comme preuve un instrument notarié et que la partie adverse affirme sous serment nourrir des soupçons au sujet de la fiabilité dudit instrument, le Sénateur et le Juge doivent convoquer le notaire qui a rédigé ledit instrument pour que celui-ci produise le registre et la minute correspondants afin de dissiper tout doute quant à l'authenticité de ladite preuve ».

${ }^{14}$ Statuti della città di Roma, éd. Camillo Re, op. cit., Livre I, Rubrique XXXII, " Quod notarius scribat substantialiter dicta testium »: "Le notaire qui met par écrit les dires des témoins doit les transcrire de manière littérale et exhaustive : il ne doit pas se contenter de dire qu'un tel a dit la même chose que tel autre ».

15 Statuti della città di Roma, éd. Camillo Re, op. cit., Livre I, Rubrique CXIII, «De notariis » : «Lorsqu'il est engagé pour rédiger un contrat ou un testament, le notaire doit, avant de quitter le lieu où s'est conclu ledit contrat, transcrire dans son registre le nom des témoins ainsi que l'essentiel du contenu de l'affaire pour laquelle il a été convoqué, afin que la vérité ne soit pas altérée par défaut de mémoire ». 
Les notaires eux-mêmes ont parfois un tel souci de l'authenticité et de la véracité des mots prononcés par leurs clients qu'ils ne les traduisent pas en latin, mais les retranscrivent directement en langue vernaculaire : ainsi, quelques actes - assez rares toutefois, même pour le $\mathrm{XV}^{\mathrm{e}}$ siècle entremêlent propos traduits en latin et phrases copiées en italien. En 1471, lorsque le comte Lorenzo de Anguillara vend des terres et des pâturages, le corps de l'acte est en italien :

Sia noto et manifesto achi la presenta polisa vedera como io. Conte Lorenzo de Anguillaria vendo et vendita faccio ad Miccinello de Cialtera 14 villoci romani herbe...

Cependant, les termes de la validation, au début et à la fin de l'acte, restent formulés en latin :

Actum in castro Ceris districtus Urbis in lovio palatii solito habitatione prefati domini Comitis Laurentii presentibus audientibus et intelligentibus hiis testibus ${ }^{16} \ldots$

Ainsi, le propos du comte apparaît comme rapporté au discours direct, renforçant par là même l'authenticité de l'acte dans son ensemble.

Ce cas constitue néanmoins l'exception et dans les faits, tous les notaires de Rome ne sont pas d'une fiabilité sans faille : certains notaires sont incapables de renseigner des informations pourtant essentielles au contrat. Parfois ce sont les confins délimitant une propriété vendue qui sont

${ }^{16}$ Roma, ASR, CNC, 1479, notaire Nicolaus Bartholomei Rosa, fo $30 \mathrm{r}^{\mathrm{o}}-31 \mathrm{r}^{\mathrm{o}}, 12$ mars 1471, acte de vente : "Acte rédigé dans le districtus Urbis, dans le castrum de Ceri, dans la loggia du palais où réside le comte Lorenzo, en présence de ces témoins, qui ont écouté et entendu ». Autres exemples d'actes rédigés partiellement en langue vernaculaire dans les archives romaines du $\mathrm{XV}^{\mathrm{e}}$ siècle : Roma, ASR, CNC, 1725, notaire Dominicus Petri de Taglientibus, $\mathrm{f}^{0} 27 \mathrm{r}^{\circ}-28 \mathrm{v}^{0}, 6$ mars 1464 ; Roma, ASR, CNC, 119, notaire Andreotius Ciottus ant., $\mathrm{f}^{\mathrm{o}} 35 \mathrm{r}^{\mathrm{o}}-36 \mathrm{r}^{\circ}$, 1er octobre 1483 ; Roma, ASR, CNC, 926, notaire Iohannes Baptista de Jays, $\mathrm{f}^{\circ} 150 \mathrm{r}^{\circ} \mathrm{v}^{\mathrm{o}}, 1484$; Roma, ASR, CNC, 706, notaire Laurentius de Festis, $\mathrm{f}^{0}$ 59bis, 15 avril 1519 ; Roma, ASR, CNC, 1443, notaire Bernardus Sabbe de Ricciis, $\mathrm{f}^{\mathrm{o}} 32 \mathrm{r}^{\circ}-35 \mathrm{r}^{\circ}, 23$ mars 1563. 
manquants ${ }^{17}$, voire dans quelques cas le prix de la transaction ${ }^{18}$ ! Plus grave encore, c'est quelquefois l'identité même des protagonistes qui est oubliée ${ }^{19}$.

Au-delà de ces défaillances humaines, tout ce système de copies successives de l'acte notarié a sans doute aussi été mis en place à la fin du Moyen Âge pour assurer une meilleure conservation des documents, un incendie étant toujours susceptible de dévorer les registres et de les réduire en cendres. Somme toute, ce que pratiquent les notaires n'est autre qu'un système de sauvegarde des données, en plusieurs exemplaires, sur plusieurs supports plus ou moins périssables (papier ou parchemin), et en des lieux diversifiés (la maison du notaire, les archives du client, les archives communales).

L'expansion quantitative de la production notariale dans la Rome du $\mathrm{XV}^{\mathrm{e}}$ siècle et la réglementation de la copie, depuis la rogatio jusqu'à l'instrument, nous renvoient également aux fréquentes contestations, falsifications de l'acte notarié et autres manigances. Les notaires romains verrouillent alors leurs documents, les protègent en les encadrant de formules de validation et d'authentification toujours plus précises.

17 Roma, ASR, CNC, 920, notaire Paulus Symonis de Jannuciis, fo 89vo $-90 \mathrm{r}^{\mathrm{o}}, 14$ mars?, location d'un terrain « extra portam Latinam in loco qui dicitur [blanc] fines [blanc] »; Roma, Archivio Storico Capitolino (ASC), Archivio Urbano, Sezione I, 254, notaire De Amatis, $\mathrm{f}^{\circ} 1 \mathrm{r}^{\circ}, 8$ janvier 1460, vente d'une vigne pour 110 florins.

18 Roma, ASR, CNC, 1172, notaire Sanctes de Odeschis de Sutro, $\mathrm{f}^{\mathrm{o}} 27 \mathrm{v}^{\mathrm{o}}-28 \mathrm{r}^{\circ}$, 16 janvier 1475, vente d'un terrain ; Roma, ASR, CNC, 920, notaire Paulus Symonis de Jannuciis, $\mathrm{f}^{0} 59 \mathrm{r}^{\circ} \mathrm{v}^{0}, 19$ novembre 1463 , vente d'une maison; $\mathrm{f}^{0} 86 \mathrm{v}^{\circ}, 16$ mars 1476 , vente d'une maison. D'une manière générale, les actes de ce notaire sont dans un mauvais état de conservation, souvent incomplets.

19 Roma, ASR, CNC, 565, notaire Philippus de Cintiis, f $6 \mathrm{r}^{\mathrm{o}} \mathrm{v}^{\mathrm{o}}, 20$ octobre 1457, location: "Magnificus vir Mathiutus de branchaleonibus de monte leono [...] locavit in perpetuum simoni [blanc] Idest quoddam ipsius casarenum situm in regione columpne...»; Roma, ASR, CNC, 1110, notaire Petrus de Meriliis, $\mathrm{f}^{\mathrm{o}} 191 \mathrm{r}^{\mathrm{o}} \mathrm{v}^{\mathrm{o}}$, 10 mai 1479 , contrat de fiançailles : «inter viros nobiles evangelistam laurentii martini de lenis de rione pinee [...] et nobilem juvenem dominum petrum filium quondam [blanc] de capranica ». 


\section{Modes traditionnels de validation des actes notariés et nouvelles pratiques d'authentification}

Dans un contexte politique et social troublé, les notaires romains renforcent les modes d'authentification traditionnels de l'acte par des pratiques inédites dont ils sont les inventeurs. La fiabilité de la minute ou de l'acte est garantie par des formules de validation, par le signum du notaire et bientôt par son sceau, mais aussi de plus en plus par le choix du lieu de l'actum et des témoins.

Tout d'abord - et je ne vais ici rien énoncer de bien neuf-, la première modalité d'authentification de l'acte reste sa datation: après l'invocation («In nomine Domini Amen»), l'acte commence par le millésime (style de la Nativité), le nom du pape régnant, l'indiction, le mois et le jour. Dans l'immense majorité des cas, le jour de la semaine et a fortiori l'heure ne sont pas mentionnés ; et lorsque le jour est mentionné, c'est toujours un samedi, jour de marchée ${ }^{20}$.

À Rome, l'usage de certains modes de validation devient systématique à partir du milieu du $\operatorname{XIV}^{\mathrm{e}}$ siècle $^{21}$ : ainsi le signum, le seing manuel du notaire, est un signe qui lui est propre. Il le trace toujours en début de registre, et parfois en bas de l'acte. Il tient lieu de souscription authentique du notaire, et se présente sous une forme géométrique, parfois parlante. Le notaire dit parfois apposer son signum, « manu signum apposui consuetum in fidem, et testimonium omnium et singulorum praemissorum »

${ }^{20}$ Roma, ASR, CNC, 1292, notaire Leonardus Petri Symonis, $\mathrm{f}^{\mathrm{o}} 216 \mathrm{r}^{\mathrm{o}} \mathrm{v}^{\mathrm{o}}, 17$ février 1481, « sabati», refutatio.

${ }^{21}$ Anna Maria Corbo, « Relazione descrittiva degli archivi notarili romani... », art. cit., p. 53 : «per quanto riguarda il simbolo che rappresenta la sottoscrizione autentica del notaio che ha redatto gli atti e che consiste a volte in una figurazione geometrica $o$ in un segno parlante, cioè il simbolo grafico del proprio nome, è da dire che non compare nei primi protocolli, ma diventa elemento costante dal 1352. Il signum veniva apposto sulla prima carta scritta, dopo l'intitulatio, e sull'ultima del protocollo. In genere la sua mancanza è dovuta alla incompletezza dei testi ». 
pour garantir la pérennité de l'acte, son authenticité «ad eternam memoriam $\gg^{22}$.

À partir du dernier quart $\mathrm{du} X \mathrm{XV}^{\mathrm{e}}$ siècle à Rome, certains notaires apposent également un sceau, très petit du reste, pour garantir certains actes. On trouve également quelques signatures autographes de certains de leurs clients ${ }^{23}$ : ainsi, dans un acte de 1471, le client rédige une ligne de sa propre main pour garantir sa validité : «Ego Laurentius manu propria me subscripti ad fidem et sigillam ${ }^{24} \gg$.

Ces éléments traditionnels de validation de l'acte notarié me paraissent bien connus et plutôt ordinaires. Je m'attarderai à présent davantage sur les dernières lignes des documents produits par les notaires, en tentant d'interpréter de manière nouvelle leur contenu.

À la fin de l'acte, dans un paragraphe bien isolé du reste du texte, se détache l'actum qui comprend le lieu et la liste des témoins. Ces dernières lignes sont fondamentales pour l'authentification de l'acte notarié ${ }^{25}$. Commençons par le lieu de signature : sa mention se fixe seulement vers la fin $\mathrm{du} \mathrm{XIV}^{\mathrm{e}}$ siècle à Rome ${ }^{26}$. Parfois, le notaire et ses clients se rendent sur

${ }^{22}$ Roma, ASR, CNC, 1896, notaire Evangelista de Bistuciis, $\mathrm{f}^{\circ} 19 \mathrm{r}^{\circ}$.

${ }^{23}$ Roma, ASR, CNC, 263, notaire Hieronymus de Brandinis, $\mathrm{f}^{\circ} 429 \mathrm{r}^{\circ}$ : signature autographe d'un Della Valle (vers 1498-1499).

24 Roma, ASR, CNC, 1479, notaire Nicolaus Bartholomei Rosa, f $30 r^{\circ}-31 r^{\circ}, 1471$ (baron Anguillara).

${ }^{25}$ Anna Maria Corbo, «Relazione descrittiva degli archivi notarili romani... », art. cit., p. 55 : «Anche la collocazione del nome dei testimoni è dapprima incerta : Paulus de Serromanis, dal 1347 al 1377, li collocava all'inizio dell'atto, dopo la data ; cosi Laurentius Johannis Stagla (1371-1372), mentre tutti i notai successivi ponevano $i$ nomi dei testimoni in calce al documento dopo l'actum. Unica eccezione Jacobus Petri Johannis Ciole, già citato, che anteponeva al testo del documento l'actum e $i$ testes. Queste anomalie potrebbero attribuirsi ad un influsso bizantino come in alcune carte dell'Italie meridionale? Non si trovano mai sottoscrizioni autografe ; $i$ nomi erano inseriti dal notaio e non mancano mai, neppure nelle imbreviature, in quanto elemento indispensabile per conferire efficacia giuridica al documento ».

${ }^{26}$ Anna Maria Corbo, « Relazione descrittiva degli archivi notarili romani... », art. cit., p. 54: «L'actum, cioè l'indicazione del luogo in cui veniva rogato il negozio giuridico, ha, come la collocazione dei testimoni, un periodo di incertezza prima di fissarsi in calce al documento. Mentre infatti nei primi protocolli notarili che si sono 
le lieu même où se trouve le bien concerné par la transaction, et qui peut être assez lointain, parfois à l'extérieur de la muraille aurélienne. Dans ce cas, puisqu'ils se sont rendus à l'endroit précis où se trouve le terrain vendu ou loué, le lieu de l'actum atteste par lui-même de l'exactitude des informations rapportées par le notaire, en particulier des informations topographiques. On l'a dit, les notaires ne sont pas toujours très fiables quant à la localisation des biens et ils commettent parfois des erreurs, surtout lorsque le terrain se situe à plusieurs dizaines de kilomètres du lieu de l'actum. Il est probable qu'ils ne se soient même jamais rendus sur les lieux... Un actum non pas Rome, mais extra urbem, est donc une garantie supplémentaire de l'authenticité de l'acte ${ }^{27}$. L'éventail de ces lieux est très varié : il me semble en outre qu'ils se diversifient au cours du $\mathrm{XV}^{\mathrm{e}}$ siècle et qu'il s'établit même une hiérarchie des lieux d'actum. Ainsi, certains endroits sont privilégiés pour certains types de contrat : pour les contrats de fiançailles par exemple, on se rendra plus volontiers dans une église, mais l'acte sera signé sous un portique s'il s'agit d'un petit prêt. Par conséquent, la solennité même du lieu de l'actum rejaillit sur le document notarié et garantit un degré d'authenticité renforcé (de même que le statut social et le nombre des témoins choisis). En outre, un lieu particulier apparaît dans la

consultati non compare affatto, lo trovo per la prima volta in un atto del 18 dicembre 1368 del notaio Paulus de Serromanis. Questo elemento è generalmente assente anche nei protocolli di Laurentius Johannis Stagla negli anni 1371-1372, ad eccezione di alcuni atti di particolare natura come $i$ matrimoni. L'actum quindi, dopo alcune incertezze, verso la fine del secolo XIV diventa regolare, con l'eccezione del notaio Jacobus Petri Johannis Ciole che ancora nel 1419 lo pone, insieme ai testes, all'inizio, dopo la data ».

${ }^{27}$ Exemples d'actes dont l'actum est extra urbem: Roma, ASR, CNC, 1104, notaire Petrus de Meriliis, $\mathrm{f}^{0} 6 \mathrm{v}^{\circ}$, $1 \mathrm{er}$ octobre 1458 (casale vocatus cicognola nova): "Actum in dicto casali et tenimento et redimento"; Roma, ASR, CNC, 1490, notaire Johannes Stephani de Rubeis, $\mathrm{f}^{\circ} 83 \mathrm{v}^{\circ}-84 \mathrm{v}^{\circ}, 26$ avril 1485, Venditio casalis La Selce, In partibus Insule, à Petrus Maximi de Maximis de rione Parione; Roma, ASR, CNC, 1082, notaire Augustinus de Martinis, f $\mathrm{f}^{\circ} 299 \mathrm{r}^{\circ}$, 6 juillet 1477, Investimentum : "Actum extra urbem in dicti casali»; Roma, ASC, AU, Sezione I, 254, notaire De Amatis, $\mathrm{f}^{\mathrm{o}} 154 \mathrm{r}^{\circ}, 29$ mai 1463, Investimentum : «Actum extra urbem in dicto loco presentibus ibidem hiis testibus videlicet...». 
seconde moitié $\mathrm{du} \mathrm{XV}^{\mathrm{e}}$ siècle à Rome : l'étude de notaire ${ }^{28}$. Choisir le bureau du notaire comme lieu de signature d'une transaction semble conférer à cette dernière un surcroît d'autorité : ainsi, dès 1468, Augustinus de Martinis rédige certains actes « in studio domu mei habitatione notarii », ou bien «in studio mey notarii $»^{29}$, et même dix ans plus tard, dans son antestudio $^{30}$, preuve que son étude s'est agrandie...

Viennent enfin les testes, les témoins : leur nombre et leur qualité varient en fonction de la nature du contrat ainsi qu'en fonction du statut social des protagonistes. $\mathrm{Au} \mathrm{XV} \mathrm{X}^{\mathrm{e}}$ siècle, ils sont toujours dits «presentibus, audientibus et intelligentibus »: «qui sont présents, qui entendent et qui comprennent ». En dernier ressort, quand toutes les informations ont été perdues - la minute et l'instrument, le notaire étant décédé -, les témoins sont seules preuves du contrat et peuvent venir en témoigner dans un procès. La présence des témoins est une manière d'authentifier l'acte qui ne passe pas par l'écrit : le contrat reste présent dans leur mémoire, comme si on avait ainsi produit dans leur esprit une forme de copie «virtuelle», latente et impalpable, de l'acte.

Les notaires romains à la fin du Moyen Âge ont renforcé le dispositif de validation de leurs écrits, produisant des copies, des « sauvegardes » à la

${ }^{28}$ Anna Maria Corbo, «Relazione descrittiva degli archivi notarili romani dei secoli XIV-XV nell'Archivio di Stato e nell'Archivio Capitolino », art. cit., p. 55 : «In questo periodo è rara l'indicazione in domo mei notarii mentre diventa sempre più frequente alla metà del secolo $X V$, quando appare chiaro che il notaio cominciava ad avere un proprio studio o ufficio dal quale si allontanava solo per atti di particolare natura, come i testamenti, o per personaggi di elevato ceto sociale o quando la parte contraente fosse una donna ».

${ }^{29}$ Par exemple : Roma, ASR, CNC, 1082, notaire Augustinus de Martinis, $\mathrm{f}^{\circ}$ 95 $\mathrm{r}^{\mathrm{o}}, 24$ mars $1468 ; \mathrm{f}^{\mathrm{o}} 104 \mathrm{r}^{\mathrm{o}}, 13$ juillet $1468 ; \mathrm{f}^{\mathrm{o}} 108 \mathrm{v}^{\mathrm{o}}-109 \mathrm{r}^{\circ}, 19$ octobre $1468 ; \mathrm{f}^{\mathrm{o}} 109 \mathrm{v}^{\mathrm{o}}, 22$ octobre $1468 ; \mathrm{f}^{\mathrm{o}} 110 \mathrm{r}^{\mathrm{o}}, 23$ octobre $1468 ; \mathrm{f}^{\mathrm{o}} 110 \mathrm{v}^{\mathrm{o}}, 28$ octobre $1468 ; \mathrm{f}^{\mathrm{o}} 113 \mathrm{v}^{\mathrm{o}}, 21$ novembre 1468. De même, le notaire Iohannes de Michaelis a un studio chez lui en 1489 au plus tard (Roma, ASR, CNC, 1135, $\mathrm{f}^{\circ} 533 \mathrm{v}^{\circ}$ : " Actum in studio domus mei notarii »); le notaire Antonius de Thomeys a un studio dans sa maison dès 1459 (Roma, ASR, CNC, 1774, $\mathrm{f}^{\circ} 19 \mathrm{r}^{\mathrm{o}} \mathrm{v}^{\mathrm{o}}, 16$ mai 1459 : «Actum Rome in rione columpne in domo mey notari videlicet in studio $»)$.

${ }^{30}$ Roma, ASR, CNC, 1082, notaire Augustinus de Martinis, $\mathrm{f}^{0} 302 \mathrm{v}^{\mathrm{o}}, 31$ août 1477 ; $\mathrm{f}^{\mathrm{o}} 303 \mathrm{r}^{\circ}, 1$ er septembre 1477 ; f $317 \mathrm{r}^{\circ}, 7$ janvier $1478 ; \mathrm{f}^{\mathrm{o}} 365 \mathrm{v}^{\circ}, 13$ mars 1480 . 
fois écrites et mémorielles, matérielles et immatérielles, et ce, de manière systématique, y compris pour de petites transactions.

\section{Étude de cas: le cas du notaire Augustinus de Martinis, prisonnier des geôles napolitaines en 1478}

Préserver l'intégrité des minutiers, se prémunir contre la fraude et la falsification de ces registres, constituent des enjeux essentiels pour le notaire. Dans la législation communale, la falsification d'actes notariés est assimilée à un vol $^{31}$ et passible de très lourdes peines : selon les statuts urbains de 1363, le notaire n'a pas le droit d'annuler, de rayer ni de supprimer de quelque manière que ce soit un acte notarié de son registre ${ }^{32}$, à moins que le client n'en fasse la requête explicite ou qu'il ne s'agisse d'un contrat extorqué sous la contrainte ou sous la torture auprès d'un citoyen croupissant en prison ${ }^{33} \ldots$ Le notaire qui produit un faux risque une

${ }^{31}$ L'article des statuts urbains de 1363 concernant la falsification d'actes notariés se situe juste avant celui punissant les voleurs, en particulier les voleurs de bétail.

${ }^{32}$ Statuti della città di Roma, éd. Camillo Re, op. cit., Livre 1, Rubrique XXXVI, «De protocollis non cancellandis»: «Non possit notarius cancellare prothocollum alicuius instrumenti nisi mandato illius vel illorum ad cuius vel quorum utilitatem factum est, et si contrafecerit in .XXV. libr. prov. puniatur et nichilominus ex tali prothocollo sic cancellato possit instrumentum mandato Iudicis relevari et publicari ».

${ }^{33}$ Statuti della città di Roma, éd. Camillo Re, op. cit., Livre 2, Rubrique XXXVII, «De instrumentis et cautionibus non valituris »: "Item statuimus et ordinamus quod si aliquis civis Romanus aliquem civem Romanum in quo iurisdictionem non habeat ceperit vel in carcerem miserit seu capi et micti fecerit et ab eo seu ab alio vel ab aliis pro eo extorserit seu extorqueri fecerit seu ipsum captum seu alium pro eo emictere fecerit cautionem cuiuscumque qualitatis seu instrumenta fieri fecerit quecumque pacta conventiones continentia, talis cautio sit ipso iure nulla, nec ex ea illi vel illis quibus facta est actio vel ius aliquod oriatur vel acquiratur contra ipsum detemptum seu alium vel alios qui pro eo cautiones huiusmodi emiserit principaliter vel accessorie, sed tam principales quam fideiuxores qui sic in cautione tali intervenerint ab omni nexu dicte cautionis liberi sint et plenissimam securitatem habeant auctoritate presentis statuti, ut nec obligati fuisse fingantur, Senator quoque ad cuiuscumque petitionem compellat illos qui in cautione huiusmodi extorserunt, seu illos quibus talis cautio facta fuit, instrumentum tale incisum reddere ac plenissimam refutationem liberationem et acceptilationem facere de predictis illi qui dictam cautionem emiserint et in nullo sibi remaneant obligati, compellat quoque notarium qui dictum instrumentum scripsit instrumentum seu prothocolla, ipsius 
amende dont le montant est compris entre 500 et 4000 livres, et doit indemniser la personne lésée. S’il ne paie pas l'amende dans les dix jours, on lui amputera la main droite. De plus, après avoir été condamné pour faux, il ne pourra plus jamais exercer le métier de notaire, et tous les instruments qu'il aura produits après cette condamnation seront tenus pour nuls et non avenus ${ }^{34}$.

C'est très vraisemblablement l'existence de telles mesures dans les statuts de Rome qui explique cette page tout à fait unique que l'on rencontre dans l'un des registres du notaire Augustinus de Martinis, datée de 1483 :

De mensibus septembris a medietate, octobris, novembris et decembris, nulli sunt contracti, quia, ad servitio reverendissimorum dominorum

instrumenti delere et totaliter cancellare, ut ex eis in perpetuum nullum possit relevari. Et si dictus Notarius non viveret tunc Iudex cause faciat dictum instrumentum seu prothocolla cassari et cancellari per correctores collegii notariorum Urbis. Et predicta fiant saltim probata publica fama per quinque bonos viros quos dictus dominus Senator vel Iudex coram quibus ageretur causa et allegaretur cautionem ipsam extortam vel emissam fore ad ipso capto vel detento vel ab alio pro eo et predicta locum habeant in preteritis presentibus et futuris aliquo capitulo supra vel infra posito non obstante. Et hoc preciso firmo manente, per quod ceteris in contrarium loquentibus volumus derogari, et predicta fiant sine strepitu et figura iudicii ordine et sollempnitate iudiciorum et iddem per omnia servetur et fiat si aliquis conquereretur se fecisse aliquem contractum per vim vel metum alicui de Urbe vel eius districtu et eadem probatio publice fame sufficiat iuxta formam suprascriptam ».

${ }^{34}$ Statuti della città di Roma, éd. Camillo Re, op. cit., Livre 2, Rubrique XXXV, «De notario faciente falzam cartam et de fieri faciente " : Notarius qui fecerit falzam cartam et qui eam fieri fecerit quilibet in solidum puniatur in Vc libris prov. si fuerit pedes. Si vero miles vel de genere militum vel cavallaroctus in mille libris prov. Si vero baro de magnatibus vel eius bastardus in IIIIm libris prov. et in quolibet casu predictorum dampnum parti lese resarceat per alterum eorumdem qui super hoc fuerit ad iudicium tractus. Quas si non soluerit infra $X$ dies a die late sententie seu postquam in fortiam Senatoris pervenerit amputetur sibi manu destra. Et postquam fuerit diffidatus seu condempnatus de falzo notarius supradictus perpetuo non possit exercere tabellionatus officium. Etiam instrumentis per eum factis post diffidationem seu condempnationem iam dictam fides nullatenus habeatur. Et locum habeat in preteritis et futuris. Et qui scienter produxerit falzum instrumentum in CC libris camere condempnetur si fuerit pedes. Si vero miles vel de genere militum vel cavallaroctus in Vc libris prov. Si vero baro de magnatibus vel eius bastardus in mille libris prov. ». 
cardinalium Sabelli et Columpne captivorum in castro Sancti Angeli, ego infelix Augustinus captus fui, ductus juxu regis Neapoliti in galea, demum positus in Castro-Novo regio, et bene custoditus ibi, denique liberatus de castro fidejussoribus de tribus milibus ducatis ${ }^{35} \ldots$

Ce qui frappe dans ce texte, hormis l'aventure rocambolesque de ce notaire, embarqué malgré lui dans le conflit qui oppose Rome à Naples, c'est le caractère personnel de cette note. On pourrait certes la rapprocher de ces petites notations historiques, rares et brèves (mort d'un pape ou d'un cardinal, inondation du Tibre...) qui ponctuent les registres notariés. Comme dans d'autres villes italiennes à la fin du Moyen Âge, à Rome les notaires endossent par moments la fonction de chroniqueur de la ville. Ils sont ainsi garants de l'authenticité historique des événements relatés avec précision : le notaire De Bertonibus rapporte ainsi un tremblement de terre qui frappe Rome le 15 mars 1477, en précisant l'heure (entre la huitième et la neuvième heure de la nuit) ${ }^{36}$. Or, Augustinus de Martinis n'a pas pour habitude de se livrer ainsi dans ses registres. La raison qui le pousse à rédiger cette confession autobiographique dans un registre est tout autre, elle a trait à l'exercice de son métier: il s'agit pour le notaire de se prémunir contre la falsification de son registre, et surtout contre d'éventuels faux instrumenta qu'on lui attribuerait à son insu. Cette page, au bas de laquelle il appose son signum pour en renforcer la validité, a pour fonction de servir de preuve contre ces potentiels faux. Si ceux-ci portent une date

${ }^{35}$ Roma, ASR, CNC, 1082, notaire Augustinus de Martinis, $\mathrm{f}^{\mathrm{0}} 400 \mathrm{r}^{\mathrm{o}}$, décembre 1483 : « À partir de la mi-septembre, et pendant les mois d'octobre, novembre et décembre, il n'y a pas eu de contrats parce que, étant au service des cardinaux Savelli et Colonna, captifs dans le château Saint-Ange, moi, pauvre Augustinus, j'ai été capturé et conduit en galère, sur ordre du roi de Naples, à Castro Novo où je fus retenu prisonnier ; en fin de compte, j'ai été libéré contre une rançon de 3000 ducats » : il rentre à Rome le 21 décembre.

${ }^{36}$ Roma, ASR, CNC, 228, notaire De Bertonibus, $\mathrm{f}^{\circ} 23 \mathrm{v}^{\circ}:$ : MEMORIA Nota Qualiter dicto die XV mensis martii 1477 Circha VIII vel novam horam noctis. In urbe fuit Terramutum...». 
comprise entre septembre et décembre 1483, il pourra ainsi prouver leur nature frauduleuse. En effet, sans ce texte, des individus malveillants pourraient l'accuser d'avoir perdu les originaux, dénonçant ainsi une mauvaise conservation des registres. La seule absence dans le cahier du notaire des minutes originales ne semble pas suffire à justifier qu'il n'y ait pas eu conclusion de contrat, et Augustinus se barde ainsi d'une garantie supplémentaire. Cette page est donc primordiale pour l'exercice de la profession notariale, car sans elle, c'est la fiabilité de l'ensemble du registre notarié qui pourrait être remise en cause...

À l'appuide mon hypothèse, je citerai une autre petite notation semblable, qu'on trouve chez le même Augustinus de Martinis, en mai 1469 : il affirme s'être rendu à Padoue, entre le 23 mai et le $1^{\text {er }}$ juillet, et ajoute que «et adeo de mense junii non extendo contractum ${ }^{37} »$. Notre notaire innove donc, en inventant une nouvelle pratique d'authentification des actes, totalement inédite. Ce qui importe donc pour garantir l'authenticité des registres notariés, c'est également l'intégrité du registre lui-même, qu'il faut préserver à tout prix. Celui-ci ne doit pas présenter de solution de continuité dans la chronologie des actes, au risque de prêter le flanc à l'accusation de mauvaise conservation et de fraude. Le souci de la continuité chronologique des actes au sein du registre est donc devenu une nouvelle garantie d'authenticité des documents.

Concluons à présent. De copistes - simples scribes pratiquant leur métier en recopiant des formulaires d'actes notariés - les notaires romains sont devenus à la fin du Moyen Âge de véritables auteurs, garants de l'autorité légale et littérale du texte. Certes, ils continuent à copier sur

${ }^{37}$ Roma, ASR, CNC, 1082, notaire Augustinus de Martinis, $\mathrm{f}^{\circ} 125 \mathrm{r}^{\circ}$ : « 1469 DIE XXIII MAII RECESSI AB URBE et petii Civitatem paduanam et non redivi usque ad primam diem mensis julii sequentis et adeo de mense junii non extendo contractum ». Il ne peut donc pas y avoir d'actes notariés datés du mois de juin 1469 . 
commande bien des textes auxquels ils apportent un surcroît d'authenticité en y apposant la mention «Copia »- ici une rubrique extraite des statuts urbains ou des statuts d'un métier ${ }^{38}$, là la copie d'une incantation pour faire tourner les esprits en rond ${ }^{39}$ ou une prophétie sur le fabuleux destin d'un roi de France $^{40} \ldots$ Cependant les notaires ne se contentent pas de recopier des formulaires «prêts-à-copier» ou extraits de textes législatifs. Comme l'écrit Odile Redon, «ils dépassent leur strict rôle professionnel en inventant des formes d'écriture ${ }^{41} »$. Ils sont de véritables auteurs, qui rédigent leurs actes à la première personne, à l'image d'Augustinus de Martinis ou du notaire qui rédigea l'attestation de «perte de virginité » de la petite Paulina, évoquée plus haut. Mieux, ils sont parfois les auteurs de récits de miracle, auxquels ils confèrent la garantie de la fides publica ${ }^{42}$. Bénéficiant du « droit d'être crus de tous », pour reprendre la belle formule de Girolamo Arnaldi ${ }^{43}$, les notaires italiens sont réellement devenus, à la fin du Moyen Âge, de véritables auteurs.

${ }^{38}$ Par exemple : Roma, ASR, CNC, 1726, notaire De Taglientibus, $\mathrm{f}^{\mathrm{0}} 74 \mathrm{r}^{\mathrm{0}}, 17$ octobre 1489, Copia: "Nos petrus de mazabufalis, prosper de Sancta Cruce et heunufrius colutie de ciecchis consules artis mercantie pannorum urbis... ».

39 Roma, ASR, Ospedale del San Salvatore ad Sancta Sanctorum, 467, n ${ }^{\circ}$ 25C: "Incantesimo per constringere li spiriti in circolo ».

40 Roma, ASR, Ospedale del San Salvatore ad Sancta Sanctorum, 467, $\mathrm{n}^{\mathrm{o}} 25 \mathrm{C}$ : "Profezia del 1423 circa le vittorie di Carlo figlio di Lodovico re di Francia ».

${ }^{41}$ Odile Redon, "Les notaires dans le paysage culturel toscan des XIII ${ }^{\mathrm{e}}-\mathrm{XIV}^{\mathrm{e}}$ siècles. Scribes, traducteurs, auteurs », dans Hommage à Jacqueline Brunet, dir. Marcella Diaz-Rozzotto, Paris, Les Belles Lettres, 1997, p. 213-222.

42 Roma, ASR, CNC, 920, notaire Paulus Symonis de Jannuciis, $\mathrm{f}^{\mathrm{o}} 9 \mathrm{r}^{\mathrm{o}} \mathrm{V}^{\mathrm{o}}, 1460$, Miraculum.

${ }^{43}$ Girolamo Arnaldi, "Il notaio-cronista e le cronache cittadine in Italia », dans La Storia del diritto nel quadro delle scienze storiche, Atti del primo congresso internazionale della società italiana di storia del diritto, Firenze, 1966, p. 293-309, p. 303. 